\title{
Influence of complex defects on electrophysical properties of GaP light emitting diodes
}

\author{
O. Konoreva $^{1}$, E. Malyj ${ }^{1}$, S. Mamykin ${ }^{2, *}$, I. Petrenko ${ }^{1}$, M. Pinkovska ${ }^{1}$, V. Tartachnyk ${ }^{1}$ \\ ${ }^{1}$ Institute for Nuclear Researches, NAS of Ukraine, \\ 47, prospect Nauky, 03028 Kyiv, Ukraine \\ ${ }^{2} V$. Lashkaryov Institute of Semiconductor Physics, NAS of Ukraine, \\ 41, prospect Nauky, 03028 Kyiv, Ukraine \\ *Corresponding author phone: +38(044)-525-37-49; e-mail: myrglory@yahoo.com
}

\begin{abstract}
In order to estimate the role of complex defects on GaP light emitting diodes (LED) operation, luminescent and electrical characteristics of GaP LEDs irradiated with reactor neutrons have been studied. It has been stated that nonradiative levels of radiation defects affect electroluminescence quenching. From the analysis of the tunnel current, the density of dislocations in the depleted part of the $p-n$ junction was obtained. Neutron induced disorder regions do not change the tunnel component of the direct current of red diodes, increasing the dislocation density, because the carrier flow along the "tunnel shunts" is blocked.
\end{abstract}

Keywords: gallium phosphide, light emitting diode, defect, luminescence and quantum yield.

Manuscript received 21.01.14; revised version received 12.05.14; accepted for publication 12.06.14; published online 30.06.14.

\section{Introduction}

The external quantum yield of light emitting diode (LED), which determines its effectiveness, depends on three factors: $\eta_{E}=\eta_{i} \eta_{l} \eta_{o}$, where $\eta_{i}$ is the coefficient of minority carrier injection, $\eta_{l}-$ effectiveness of light generation and $\eta_{o}$ - coefficient of light output. The first and second multipliers characterize the quality of the crystal and $p-n$ junction and are usually combined as the internal quantum yield $\eta_{I}=\eta_{i} \eta_{l}[1]$.

It is more difficult to control a commercial device than a separate crystal, especially if complex defects such as dislocations, radiation induced defects and other ones are available and dominate in device properties. One should know that defects of "dark line" and "dark spot" types, responsible for degradation processes in
$\mathrm{GaP}$ LEDs, are caused by the accumulation of dislocation networks [2].

While creating the $p-n$ junction in LEDs, layers of different conductivity are deposited epitaxially on substrate. Due to the interface between these two films, defects are accumulated within the depleted region of transition at a distance of 2-3 diffusion lengths from this interface. It negatively affects the value of the internal quantum yield.

The situation is still worsening due to the growth of dislocations out of the substrate surface, which is previously polished and possesses a great amount of line-type defects $\left(\rho>10^{6} \mathrm{~cm}^{-2}\right)$. In order to eliminate the influence of the substrate surface, it is unreasonable to increase the $n$-film thickness, because self-absorption of photons will increase. 
Therefore, we hope that the study of behavior of dislocations in the transition region will help to predict the LED performance.

The authors of the papers [3, 4] showed the possibility of appearance of the excess direct tunnel current not only in degenerate $p-n-n$ junctions and heterostructures. It turns out that the model of "dislocation shunt", which describes interdefect tunneling of carriers in the space charge region, can be applied to the homojunction where the tunneling process occurs along the dislocation line.

Based on the conclusions obtained in $[3,4]$, one can appreciate the density of dislocations in the $p-n$ junction and identify the impact of extreme external factors (including also fast particle irradiation - in this case) on interdislocation tunneling of carriers.

We have presented the results of the study of electroluminescence spectra and electrophysical characteristics of GaP LEDs irradiated with fast reactor neutrons. Current-voltage characteristics of initial diodes and those with radiation defects have been analyzed using the method proposed in the paper [4], and the dislocation influence has been estimated as well.

\section{Experimental}

We studied green and red industrial LEDs made using double solid epitaxial methods. Green LEDs were doped with nitrogen atoms, red - with zinc and oxygen, simultaneously. The electron concentration was equal to $2 \cdot 10^{17} \mathrm{~cm}^{-3}$, and that of holes $-(4-5) \cdot 10^{16} \mathrm{~cm}^{-3}$. The $n$-GaP substrate was made using the Czochralsky method with the carrier concentration $(5-7) \cdot 10^{17} \mathrm{~cm}^{-3}$. The sample dimensions were $1 \times 1 \mathrm{~mm}$. The diodes were irradiated with fast neutrons in reactor at room temperature. Current-voltage characteristics were measured manually and by the automatic system within the range $1 \ldots 10^{-9} \mathrm{~A}$ for current and 1 to $30 \mathrm{~V}-$ for voltage at temperatures ranging from 77 up to $300 \mathrm{~K}$.

Electroluminescence spectra were measured by a spectrometer BLK-C F1000-VIS NIR-1 (StellarNet Inc) for $190 \ldots 850 \mathrm{~nm}$ intervals within the same temperature range 77 to $300 \mathrm{~K}$.

\section{Result and discussion}

The electroluminescence spectra of green GaP LEDs measured at room and nitrogen temperatures are given in Fig. 1. As one can see, the low temperature spectrum possesses a fine structure due to izoelectron nitrogen impurity. In the near-edge part of the spectrum, there is a line associated with exciton bound to nitrogen atom. Its intensity is significantly weaker than luminosity of exciton bound to the pair of $\mathrm{NN}_{i}$ centers due to the lower binding energy and effect of self-absorption. When the sample temperature increases up to $300 \mathrm{~K}$, the fine structure of the spectrum disappears.

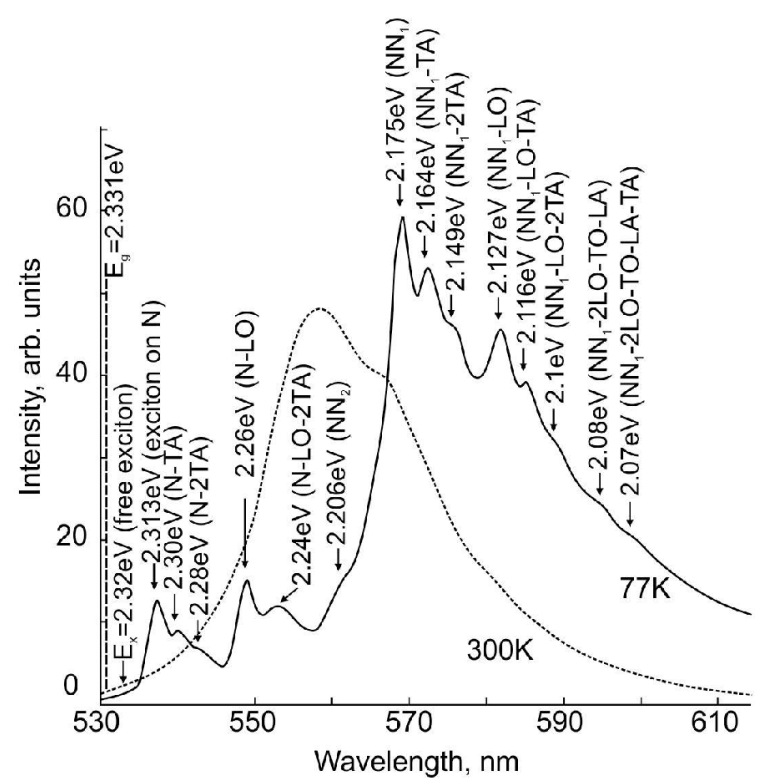

Fig. 1. Electroluminescence spectrum of GaP LED at 300 and $77 \mathrm{~K}$.

The main emission band is related with the pair $\mathrm{NN}_{1}$. It has $E=2.22 \mathrm{eV}$ and $\mathrm{LA}$ line as phonon duplication.

Irradiation of GaP LED with fast reactor neutrons causes a monotonic intensity drop of all the lines, and the luminosity of exciton bound to the isolated nitrogen atom is the most sensitive region of the spectrum to introduction of radiation defects.

It was stated in [5] and confirmed later by us and other authors [6-9] that the radiationless level of radiation defects is a reason for electroluminescence quenching.

It should be said that impurity levels existing in the forbidden band of non-irradiated diode can also be the centers of nonradiative recombination and reduce the internal quantum yield.

It was found [2] that the low value of the internal quantum yield of irradiated GaP LEDs was due to deep levels caused mainly by dislocations in the $p-n$ junction, the thickness of which was of the order of the diffusion length inherent to minority carriers.

Excess tunneling through the depleted region of the diode is a component of the injection current and can exist not only in the degenerate structures. Even at base moderate doping of the $p-n$ junction (order $\sim 10^{17} \mathrm{~cm}^{-3}$ ), this opportunity may be provided by hopping-like tunneling through the space charge region $[3,4]$.

In the dislocation shunt model, the exponential part of the current-voltage characteristics is described as follows:

$$
I=I_{0}\left(\exp \frac{e V}{\varepsilon}-1\right)
$$


Here, unlike the Shockley formula, $I_{0}$ is the function not only of diffusion and electric constants of the base:

$$
I_{0}=e \rho v_{\mathrm{D}} \exp \left(-\frac{e V_{c}}{\varepsilon}\right),
$$

where $\rho$ is the dislocation density, $\varepsilon$ - characteristic energy, $v_{\mathrm{D}}$ - Debye frequency, and $e$ - electron charge.

The contact potential difference $V_{c}$ (like to the classical model) may be given in the form of

$$
\begin{aligned}
& V_{c}=\frac{k T}{e}\left[\ln \frac{p_{p}}{n_{i}}+\ln \frac{n_{n}}{n_{i}}\right]=\frac{k T}{e}\left[\ln \frac{p_{p} n_{n}}{n_{i}^{2}}\right]= \\
& =\frac{k T}{e} \ln \left[\frac{p_{p} n_{n}}{N_{C} N_{V} e-\frac{E_{g}}{k T}}\right]=\frac{E_{g}}{e}-\frac{k T}{e}\left\{\ln \frac{N_{C}}{n_{n}}+\ln \frac{N_{V}}{p_{p}}\right\},
\end{aligned}
$$

where $E_{g}$ is the band gap; $N_{C}, N_{V}$ are densities of states in the appropriated conduction and valence bands; $n_{n}, p_{p}$ are concentrations of electrons and holes, respectively.

Considering the $\mathrm{GaP}$ temperature dependence of the band gap $E_{g}=E_{g}(0)-\alpha T$, where $\alpha$ is a constant, one can define the $\varepsilon$ parameter and dislocation density:

$\rho=\frac{I_{0}}{e v_{\mathrm{D}}}\left(\frac{n_{n} p_{p}}{N_{C} N_{V}}\right)^{\frac{k T}{g}} \exp \frac{E_{g}}{\varepsilon}$.

$\mathrm{GaP}$ current-voltage characteristics of green (active region is doped with nitrogen), red (doped with both zinc and oxygen) and the same diode irradiated with fast reactor neutrons $\left(E=1 \mathrm{MeV}, F=10^{16} \mathrm{~cm}^{-2}\right)$ are given in Fig. 2.

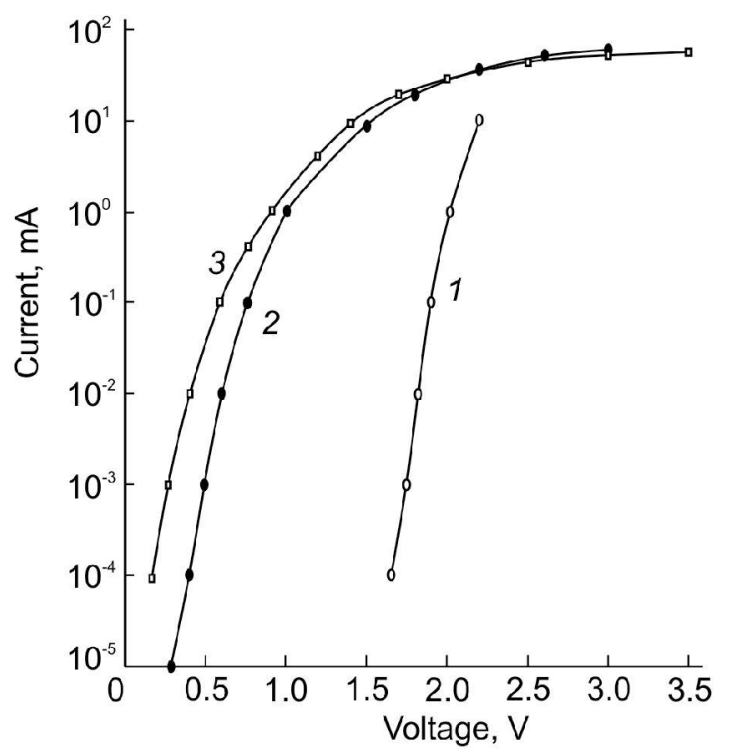

Fig. 2. Current-voltage characteristics of GaP LED diode: irradiated with fast reactor neutrons (1); initial red (2) and green (3) diodes.
In the latter case, the neutron dose was so high that the diode stopped emitting even at significant levels of injection $(I=50 \ldots 60 \mathrm{~mA})$. By extrapolating the exponential plot of the current-voltage dependence on the zero displacement, it is possible to obtain $I_{0}$, where $\varepsilon$ is the curve slope. Measurements carried out within the range $77 \ldots 300 \mathrm{~K}$ showed that both the parameters did not practically depend on temperature. Considering that $N_{V}=N_{C}=8 \cdot 10^{18} \mathrm{~cm}^{-3}$ and $n \approx p \approx 10^{17} \mathrm{~cm}^{-3}$, corresponding to the mean value of the carrier concentration in the base of the diodes of AL-102 BM series, the average dislocation density in green $\mathrm{GaP}(\mathrm{N})$ is equal to $\rho_{\mathrm{D}}=7.5 \cdot 10^{10} \mathrm{~cm}^{-2}$, in red GaP $(\mathrm{Zn}-\mathrm{O}) \rho_{\mathrm{D}}=$ $4 \cdot 10^{8} \mathrm{~cm}^{-2}$.

After neutron irradiation of red diodes, $\rho_{\mathrm{D}}$ is equal to $4.4 \cdot 10^{8} \mathrm{~cm}^{-2}$.

So, it is obvious that in red diodes neutrons do not appreciably affect the density of linear defects dislocations, despite the fact that thermal wedges and associated deformation fields or Brinkman peaks of displacements appear in irradiated objects.

It is also obvious that under neutron irradiation the crystal structure becomes so disordered that tunnel jumps, occuring in non-irradiated crystal along the dislocation tubes, become impossible due to fractures of dislocation lines. An additional increase of dislocation densities in the periphery areas of disordered regions does not affect the value of the tunnel component of the direct current. "Dislocation shunt" does not work within the space charge region.

\section{Conclusions}

Main lines of electroluminescence spectra of green $\mathrm{GaP}(\mathrm{N})$ LEDs have been identified at different temperatures. At $77 \mathrm{~K}$, the thin structure was discovered with the peak associated with exciton bound to separate nitrogen atom. Its intensity is significantly weaker than that of exciton bound to the pair of NN centers due to the lower binding energy and effect of self-luminosity. The structure vanishes when temperature increases up to $300 \mathrm{~K}$. Neutron irradiation causes monotonic quenching of all the components in the electroluminescence spectrum.

The model of dislocation tunneling makes it possible to assess the density of dislocations in the depleted part of the $p-n$ junction. This value $\rho_{\mathrm{D}}$ is close to $7.5 \cdot 10^{10} \mathrm{~cm}^{-2}$ in green $\operatorname{GaP}(\mathrm{N})$ and in red $\mathrm{GaP}$ $(\mathrm{Zn}-\mathrm{O})-4 \cdot 10^{8} \mathrm{~cm}^{-2}$. Neutron irradiation does not significantly affect $\rho_{\mathrm{D}}$ of red diodes.

It has been suggested that the disorder regions induced with neutrons are able to block the current flow along the "tunnel shunts".

The authors thank to M. Lytovchenko for his help in carrying out the experiment. 


\section{References}

1. F.E. Shubert, Light Emitting Diodes, Fizmatlit Publ., Moscow, 2008 (in Russian).

2. A. Berg, P. Din, Light Emitting Diodes, Mir Publ., Moscow, 1979 (in Russian).

3. V.V. Evstropov, Yu.V. Zhiliayev, M. Dzhumaeva, N. Nazarov, Tunnel-excess current in nondegenerated barrier p-n and m-s silicon-containing III-V compound semiconductor structures // Fizika i tekhnika poluprovodnikov, 31(2), p. 152-158 (1997), in Russian.

4. V.V. Evstropov, Yu.V. Zhiliayev, M. Dzhumaeva, N. Nazarov, A.A. Sitnikova, L.M. Fedorov, Dislocation origin and a model of the excessive tunnel current in GaP p-n structure // Fizika i tekhnika poluprovodnikov, 34(11), p. 1357-1368 (2000), in Russian.

5. D.V. Lang, L.C. Kimerling, Observation of a thermal defect annealing in $\mathrm{GaP} / /$ Appl. Phys. Lett. 28, p. 248 (1976).
6. A. Borsakovskyj, O. Gontaruk, V. Kochkin, P. Litovchenko, V. Opilat, I. Petrenko, V Tartachnyk, Radiation influence on characteristics of GaP light emitting diodes // Functional Materials, 16, p. 313-318 (2009).

7. A.V. Gomonnai, D.B. Goyer, O.O. Goushcha, Yu.M. Azhniuk, I.G. Megela, M. Kranjcec, Radiative recombination in electron-irradiated $\mathrm{GaP}$ crystals // J. Optoelectron. Adv. Mater. 5(3), p. 641-646 (2003).

8. T. Endo, T. Nichimura, K. Nakakuki, M. Kitamura, K. Sugijama, DLTS study for energy-broadening of the defect level on introducing radiation damage in GaP // Jpn. J. Appl. Phys. 27(11), p. 2107-2112 (1988).

9. T. Endo, Yu. Hirosaki, E. Uchida, H. Miyake, K. Sugiyama, Deep levels in electron-irradiated $\mathrm{GaP}$ at $10 \mathrm{MeV} / /$ Jpn. J. Appl. Phys. 28(10), p. $1864-1870$ (1989). 